Research Article

\title{
New Tseng-Degree Gradient Method in Variational Inequality Problem
}

\author{
Zhuang Shan, ${ }^{1}$ Lijun Zhu $\mathbb{D D}^{2,3}$ Long He, ${ }^{1}$ Danfeng Wu, ${ }^{1}$ and Haicheng Wei ${ }^{4}$ \\ ${ }^{1}$ School of Mathematics and Information Science, North Minzu University, Yinchuan 750021, China \\ ${ }^{2}$ The Key Laboratory of Intelligent Information and Data Processing of NingXia Province, North Minzu University, \\ Yinchuan 750021, China \\ ${ }^{3}$ Health Big Data Research Institute of North Minzu University, Yinchuan 750021, China \\ ${ }^{4}$ Advanced Intelligent Perception Control Technology Innovative Team of NingXia, Minzu University, Yinchuan 750021, China
}

Correspondence should be addressed to Lijun Zhu; zhulijun1995@yahoo.com

Received 17 May 2020; Accepted 27 June 2020; Published 5 August 2020

Guest Editor: Zaoli Yang

Copyright (c) 2020 Zhuang Shan et al. This is an open access article distributed under the Creative Commons Attribution License, which permits unrestricted use, distribution, and reproduction in any medium, provided the original work is properly cited.

This paper focuses on the problem of variational inequalities with monotone operators in real Hilbert space. The Tseng algorithm constructed by Thong replaced a high-precision step. Thus, a new Tseng-like gradient method is constructed, and the convergence of the algorithm is proved, and the convergence performance is higher.

\section{Introduction}

Let $H$ be the real Hilbert space, and $\langle\cdot, \cdot\rangle$ and $\|\cdot\|$ are respectively defined as the inner product and norm of space $H$. Let $C$ be a nonempty closed convex subset of space $H$. Let $\mathfrak{T}$ : $H \longrightarrow H$ be an operator on the set $C$. The variational inequality problem (VIP) means to find a point $x^{*}$ on $C$ such that

$$
\left\langle\mathfrak{T} x^{*}, x-x^{*}\right\rangle \geq 0, \quad \forall x \in C .
$$

We use $V I(\mathfrak{I}, C)$ to denote the solution set of problem (1). At present, the regular method and the projection method are the two main methods to solve the problem of variational inequality. Variational inequalities can also solve other problems such as equilibrium, fixed points, and optimization. In addition, it is also widely used in the industry, especially supply chain management and transportation. Supply chain management is a new type of management concept, which emphasizes the rapid market demand response, combat readiness management, high flexibility, low risk, cost-effectiveness, and other goals, attracting many theoretical and practical industry people to study and practice it. Some well-known international companies, such as Hewlett-Packard and Dell, have made great achievements in the practice of supply chain management. Therefore, the supply chain is an effective way for enterprises to adapt to global competition in the 21st century (see eg., [1-11].

At present, the theoretical support of the variational inequality problem is gradually maturing, and people are gradually changing from the theoretical research of the problem to the construction of the variational inequality algorithm. In recent years, the variational inequality algorithm has developed rapidly, but the use conditions are harsh. The use of the Tseng algorithm does not require too harsh conditions, such as the strongly monotone or inverse strongly monotone of the problem and only one projection equation is used to approximate the solution of the problem. The specific method is as follows:

$$
\left\{\begin{array}{l}
y_{n}=P_{C}\left(x_{n}-\rho \mathfrak{T} x_{n}\right) \\
x_{n+1}=y_{n}-\rho\left(\mathfrak{T} y_{n}-\mathfrak{T} x_{n}\right) .
\end{array}\right.
$$

There $\rho \in(0,1 / L)$ and $P_{C}$ is a projection operator in set $C$ of Hilbert space $H$. In recent years, the Tseng algorithm has been widely recognized. Subsequently, Duong Viet and others improved the Tseng method. For the specific method, please see Lemma 7 [12]. 
Although the Tseng algorithm constructed by Duong Viet et al. has many advantages, the shortcoming is that the step size is too simple. The work of this paper is to improve the convergence of the algorithm by optimizing the step size, making it more precise and practical on the basis of convergence. The step size adopted in this article is currently commonly used, and it has high accuracy itself, which can effectively improve the convergence speed of the algorithm. In addition, this paper popularizes the application field of the algorithm to make it more applicable.

The structure of this paper is as follows: In Section 2, we give some necessary definitions and lemmas. In Section 3, we give an algorithm for construction. We analyze and prove that the algorithm weakly converges to a certain point in $V I$ $(C, \mathfrak{T})$ and give some inferences.

\section{Preliminaries}

Let $H$ be a real Hilbert space and $C$ be a nonempty closed convex subset of $H$. As we all know, the following equation can be directly proved in Hilbert space.

When $\forall x \in H, y \in H$, and $\alpha \in R$, we can get

$$
\begin{gathered}
\|x+y\|^{2}=\|x\|^{2}+2\langle x, y\rangle+\|y\|^{2} \\
\|\alpha x+(1-\alpha) y\|^{2}=\alpha\|x\|^{2}+(1-\alpha)\|y\|^{2}-\alpha(1-\alpha)\|x-y\|^{2} .
\end{gathered}
$$

Definition 1. Let $\mathfrak{I}$ be a operator on a Hilbert space, and then the operator $\mathfrak{T}$ is a nonexpansive operator, if the operator $\mathfrak{I}$ has the property

$$
\|\mathfrak{T} x-\mathfrak{T} y\| \leq c\|x-y\|, \quad \text { for all } x, y \in C,
$$

and for some constant $c \in[0,1]$.

Let $P_{C}$ be a projection operator in set $C$ of Hilbert space $H$, then $\forall x \in H$. There is only one point $P_{C} x$ such that $\left\|P_{C} x-y\right\| \leq\|x-y\|, \forall y \in C$. It is easy to prove that $P_{C}$ is a nonexpansive operator.

Lemma 1 (see [13]). Let $H$ be a real Hilbert space and $C$ be a nonempty closed convex subset of $H$. For a given $x \in H, z \in C$. Then, $\forall y \in C$ has the following relationship:

$$
z=P_{C} x \Longleftrightarrow\langle x-z, z-y\rangle \geq 0 .
$$

Lemma 2 (see [13]). Let $H$ be a real Hilbert space and $C$ be a nonempty closed convex subset of $H, x \in H$, then $\forall y \in C$. The following equation holds

$$
\begin{aligned}
& \text { (i) }\left\|P_{C} x-P_{C} y\right\|^{2} \leq\left\langle P_{C} x-P_{C} y, x-y\right\rangle, \\
& \text { (ii) }\left|\left\langle P_{C}, x-y\right\rangle\right|^{2} \leq\|x-y\|^{2}+\left\|P_{C} x-x\right\|^{2} .
\end{aligned}
$$

If the reader wants to know more about the nature of projections, please refer to part 3 in [13].

$$
\begin{aligned}
& \text { Definition 2. Let } \mathfrak{T}: H \longrightarrow H \text {, if } \forall x, y \in H \text { satisfies } \\
& \qquad\|\mathfrak{I} x-\mathfrak{I} y\| \leq S\|x-y\|, \quad S>0 .
\end{aligned}
$$

Then, the operator $\mathfrak{I}$ is called S-Lipschitz continuous;

$$
\langle\mathfrak{T} x-\mathfrak{I} y, x-y\rangle \geq 0,
$$

Then, the operator $\mathfrak{T}$ is called monotonic.

Lemma 3 (see [14]). Let $\left\{\varphi_{n}\right\},\left\{\delta_{n}\right\}$, and $\left\{\alpha_{n}\right\}$ be sequences in $[0,+\infty)$ such that

$$
\varphi_{n+1} \leq \varphi_{n}+\alpha_{n}\left(\varphi_{n}-\varphi_{n-1}\right), \quad \forall n \geq 1, \sum_{n=1}^{+\infty} \delta_{n}<+\infty
$$

and there is a real number $\alpha$, for any $n \in N$, such that $0 \leq \alpha_{n} \leq \alpha<1$. Then,

(i) $\sum_{n=1}^{+\infty}\left[\varphi_{n}-\varphi_{n-1}\right]_{+}<+\infty$,

where $\left[\varphi_{n}-\varphi_{n-1}\right]_{+}=\max \left\{\varphi_{n}-\varphi_{n-1}, 0\right\}$.

(i) There is a point $\varphi^{*} \in[0,+\infty)$ such that $\lim _{n \longrightarrow+\infty} \varphi_{n}=\varphi^{*}$.

Lemma 4 (Opial 1967). Let $\varnothing \neq C \subset H$ and $\left\{x_{n}\right\}$ be the sequence in $H$ such that the following two conditions hold:

(i) $\forall x \in C, \lim _{n \longrightarrow+\infty}\left\|x_{n}-x\right\|$ exist;

(ii) Every point of weakly gathering in the sequence $\left\{x_{n}\right\}$ is in $C$.

Then, $x_{n}$ weakly converges to a point $x$ in $C$.

Lemma 5 (minty, please refer to reference [15], Lemma 7.1.7). Let $\mathfrak{T}: C \longrightarrow H$ be a monotone continuous operator. Then, $x^{*}$ is the solution to problem (1) if and only if $x^{*}$ is a solution to the following problem:

$$
\text { find } x \in C \text { such that }\langle\mathfrak{I} y, y-x\rangle \geq 0, \quad \forall y \in C \text {. }
$$

Remark 1. The solution set $\operatorname{VI}(C, \mathfrak{I})$ of variational inequality (1) is closed and convex.

Lemma 6 (see [15]). Let $\mathfrak{T}: H \longrightarrow H$ be monotone and Lipschitz continuous on $H$ with the constant $S$ and $V I(C, \mathfrak{I}) \neq \varnothing, \rho$ be a positive obtained real number such that $\rho \in(0,(1 / S))$. If $\left\{\eta_{n}\right\}$ is a nonincreasing sequence, make $0 \leq \eta_{n} \leq \eta$ and

$$
\eta<\frac{\sqrt{1+8 \mathfrak{K}}-1-2 \mathfrak{\Re}}{2(1-2 \mathfrak{\Re})}
$$

where $\mathfrak{K}:=((1-\rho S) /(1+\rho S))$, then the sequence $\left\{x_{n}\right\}$ obtained by next algorithm weakly converges to $\operatorname{VI}(C, \mathfrak{I})$.

(i) Step 0: given $\rho>0$; Let $x_{0}, x_{1} \in H$ be arbitrary

(ii) Step 1: set $\omega_{n}=x_{n}+\eta_{n}\left(x_{n}-x_{n-1}\right)$ and compute

$$
y_{n}=P_{C}\left(\omega_{n}-\rho \mathfrak{T} \omega_{n}\right)
$$

When $y_{n}=\omega_{n}$, then stop iteration, and $y_{n}$ is the solution to the (VIP) problem; otherwise,

(iii) Step 2: compute 


$$
x_{n+1}=y_{n}-\rho\left(\mathfrak{I} y_{n}-\mathfrak{I} \omega_{n}\right) .
$$

Set $n=n+1$ and go to Step 1 .

Lemma 7 (see [15]). Suppose that $\rho \in(0,(1 / S))$. Let $\left\{x_{n}\right\}$ be a sequence in $H$ defined by

$$
\left\{\begin{array}{l}
x_{0} \in H, \\
y_{n}=P_{C}\left(x_{n}-\rho \mathfrak{T} x_{n}\right), \\
x_{n+1}=y_{n}-P_{C}\left(\rho \mathfrak{I} y_{n}-\rho \mathfrak{I} x_{n}\right) .
\end{array}\right.
$$

Then, the sequence $\left\{x_{n}\right\}$ converges weakly to an element of $V I(C, \mathfrak{I})$.

\section{Main Results}

Let $\mathfrak{T}: H \longrightarrow H$ be monotone and Lipschitz continuous on $H$ with the constant $S$ and $V I(C, \mathfrak{I}) \neq \varnothing$. First, we introduce Algorithm 1.
Lemma 8. Let $\left\{\rho_{n}\right\}$ be a sequence obtained by Algorithm 1, then the sequence $\left\{x_{n}\right\}$ decreases monotonically and its lower bound is $\min \left\{(\mu / S), \rho_{0}\right\}$, where $S>0$ is the Lipschitz condition constant.

Proof. According to the definition, the sequence $\left\{\rho_{n}\right\}$ is monotonically nonincreasing, because $\mathfrak{T}$ is a Lipschitz condition function. At that time, $\mathfrak{I} y_{n}-\mathfrak{T} \omega_{n} \neq 0$, and we have

$$
\frac{\mu\left\|y_{n}-\omega_{n}\right\|}{\left\|\mathfrak{I} y_{n}-\mathfrak{I} \omega_{n}\right\|} \geq \frac{\mu\left\|y_{n}-\omega_{n}\right\|}{S\left\|y_{n}-\omega_{n}\right\|}=\frac{\mu}{S} .
$$

Obviously, the sequence $\left\{\rho_{n}\right\}$ has a lower bound $\min \left\{(\mu / S), \rho_{0}\right\}$.

Theorem 1. Let the sequence $\left\{x_{n}\right\}$ be obtained by Algorithm 1 , then $\forall p \in V I(C, \mathfrak{I})$ have

$$
\left\|x_{n+1}-p\right\|^{2} \leq\left\|\omega_{n}-p\right\|^{2}-\left(1-\rho_{n}^{2} \frac{\mu^{2}}{\rho_{n+1}^{2}}\right)\left\|y_{n}-\omega_{n}\right\|^{2} .
$$

Proof

$$
\begin{aligned}
\left\|x_{n+1}-p\right\|^{2}= & \left\|y_{n}-\rho_{n}\left(\mathfrak{I} y_{n}-\mathfrak{I} \omega_{n}\right)-p\right\|^{2} \\
= & \left\|y_{n}-p\right\|^{2}+\rho_{n}^{2}\left\|\mathfrak{I} y_{n}-\mathfrak{I} \omega_{n}\right\|^{2}-2 \rho_{n}\left\langle y_{n}-p, \mathfrak{I} y_{n}-\mathfrak{T} \omega_{n}\right\rangle \\
= & \left\|\omega_{n}-p\right\|^{2}+\left\|\omega_{n}-y_{n}\right\|^{2}+2\left\langle y_{n}-p, \mathfrak{I} y_{n}-\mathfrak{I} \omega_{n}\right\rangle+\rho_{n}^{2}\left\|\mathfrak{I} y_{n}-\mathfrak{I} \omega_{n}\right\|^{2}-2 \rho_{n}\left\langle y_{n}-p, \mathfrak{I} y_{n}-\mathfrak{I} \omega_{n}\right\rangle \\
= & \left\|\omega_{n}-p\right\|^{2}+\left\|\omega_{n}-y_{n}\right\|^{2}-2\left\langle y_{n}-\omega_{n}, y_{n}-\omega_{n}\right\rangle+2\left\langle y_{n}-\omega_{n}, y_{n}-p\right\rangle+\rho_{n}^{2}\left\|\mathfrak{I} y_{n}-\mathfrak{T} \omega_{n}\right\|^{2} \\
& -2 \rho_{n}\left\langle y_{n}-p, \mathfrak{T} y_{n}-\mathfrak{T} \omega_{n}\right\rangle \\
= & \left\|\omega_{n}-p\right\|^{2}-\left\|\omega_{n}-y_{n}\right\|^{2}+2\left\langle y_{n}-\omega_{n}, y_{n}-p\right\rangle+\rho_{n}^{2}\left\|\mathfrak{I} y_{n}-\mathfrak{I} \omega_{n}\right\|^{2}-2 \rho_{n}\left\langle y_{n}-p, \mathfrak{I} y_{n}-\mathfrak{T} \omega_{n}\right\rangle .
\end{aligned}
$$

Since $y_{n}=P_{C}\left(\omega_{n}-\rho_{n} \mathfrak{T} \omega_{n}\right)$, so

$$
\left\langle y_{n}-\omega_{n}+\rho_{n} \mathfrak{T} \omega_{n}, y_{n}-p\right\rangle \leq 0,
$$

$$
\left\langle y_{n}-\omega_{n}, y_{n}-p\right\rangle \leq-\rho_{n}\left\langle\mathfrak{I} \omega_{n}, y_{n}-p\right\rangle,
$$

or

can be obtained from (16) and (18):

$$
\begin{aligned}
\left\|x_{n+1}-p\right\|^{2} \leq & \left\|\omega_{n}-p\right\|^{2}-\left\|\omega_{n}-y_{n}\right\|^{2}-2 \rho_{n}\left\langle\mathfrak{T} \omega_{n}, y_{n}-p\right\rangle \\
& +\rho_{n}^{2}\left\|\mathfrak{T} y_{n}-\mathfrak{T} \omega_{n}\right\|^{2}-2 \rho_{n}\left\langle y_{n}-p, \mathfrak{I} y_{n}-\mathfrak{T} \omega_{n}\right\rangle \\
= & \left\|\omega_{n}-p\right\|^{2}-\left\|\omega_{n}-y_{n}\right\|^{2}+\rho_{n}^{2}\left\|\mathfrak{T} y_{n}-\mathfrak{T} \omega_{n}\right\|^{2}-2 \rho_{n}\left\langle y_{n}-p, \mathfrak{T} y_{n}\right\rangle \\
\leq & \left\|\omega_{n}-p\right\|^{2}-\left\|\omega_{n}-y_{n}\right\|^{2}+\rho_{n}^{2} \frac{\mu^{2}}{\rho_{n+1}^{2}}\left\|y_{n}-\omega_{n}\right\|^{2} \\
& \quad-2 \rho_{n}\left\langle y_{n}-p, \mathfrak{T} y_{n}-\mathfrak{T} p\right\rangle-2 \rho_{n}\left\langle y_{n}-p, \mathfrak{T} p\right\rangle \\
\leq & \left\|\omega_{n}-p\right\|^{2}-\left(1-\rho_{n}^{2} \frac{\mu^{2}}{\rho_{n+1}^{2}}\right)\left\|y_{n}-\omega_{n}\right\|^{2} .
\end{aligned}
$$


(i) Step 0: given $\rho_{0}>0$. Let $x_{0}, x_{1} \in H$ be arbitrary and $\mu \in((1 / \sqrt{2}), 1)$

(ii) Step 1: set $\omega_{n}=x_{n}+\eta_{n}\left(x_{n}-x_{n-1}\right)$ and compute $y_{n}=P_{C}\left(\omega_{n}-\rho_{n} \mathfrak{T} \omega_{n}\right)$

(iii) When $y_{n}=\omega_{n}$, then stop iteration, and $y_{n}$ is the solution to the (VIP) problem; otherwise,

(iv) Step 2: compute $x_{n+1}=y_{n}-\rho_{n}\left(\mathfrak{I} y_{n}-\mathfrak{T} \omega_{n}\right)$

(v) and $\rho_{n+1}= \begin{cases}\min \left\{\left(\mu\left\|y_{n}-\omega_{n}\right\| /\left\|\mathfrak{T} y_{n}-\mathfrak{T} \omega_{n}\right\|\right), \rho_{n}\right\}, & \mathfrak{T} y_{n}-\mathfrak{T} \omega_{n} \neq 0, \\ \rho_{n}, & \text { other }\end{cases}$

(vi) Set $n=n+1$ and go to Step 1

Algorithm 1: New Tseng-degree gradient algorithm.

Theorem 2. Let $\left\{\rho_{n}\right\}$ be a sequence generated by Algorithm 1 . If $\left\{\eta_{n}\right\}$ is a nonincreasing sequence, make $0 \leq \eta_{n} \leq \eta$ and

$$
\eta<\frac{\sqrt{1+8 \mathfrak{K}}-1-2 \mathfrak{\Re}}{2(1-2 \mathfrak{K})},
$$

where $\mathfrak{\Re}:=1-\mu^{2}$, then the sequence $\left\{x_{n}\right\}$ obtained by Algorithm 1 weakly converges to $\operatorname{VI}(C, \mathfrak{T})$.

Proof. The first step.

$$
\text { Let } \quad \boldsymbol{R}_{n}:=\left(1-\rho_{n}^{2}\left(\mu^{2} / \rho_{n+1}^{2}\right) / 1+\rho_{n}^{2}\left(\mu^{2} / \rho_{n+1}^{2}\right)\right)
$$
$\leq 1-\rho_{n}^{2}\left(\mu^{2} / \rho_{n+1}^{2}\right) \leq 1-\mu^{2}=\mathfrak{\Omega}$.

Since $\left(\sqrt{1+8 \mathfrak{K}_{n}}-1-2 \mathfrak{\Re}_{n}\right) /\left(2\left(1-2 \mathfrak{\Re}_{n}\right)\right)$ monotonically increases in $(0,(1 / 2)) ;\left\{\rho_{n}\right\}$ monotonically decreases, so $\left(\rho_{n}^{2} / \rho_{n+1}^{2}\right) \geq 1$, and then there $\eta<\left(\left(\sqrt{1+8 \mathfrak{\Omega}_{n}}-\right.\right.$ $\left.\left.1-2 \mathfrak{\Re}_{n}\right) / 2\left(1-2 \mathfrak{\Re}_{n}\right)\right)<\left((\sqrt{1+8 \mathfrak{K}}-1-2 \mathfrak{\Re}) / 2\left(1-2 \mathfrak{\Re}_{n}\right)\right)$.

From the second step, through the construction of $x_{n+1}$, we can get

$$
\begin{aligned}
\left\|x_{n+1}-y_{n}\right\| & =\left\|y_{n}-\rho_{n}\left(\mathfrak{T} y_{n}-\mathfrak{I} \omega_{n}\right)-y_{n}\right\| \\
& \leq \rho_{n}\left\|\mathfrak{I} y_{n}-\mathfrak{T} \omega_{n}\right\| \\
& \leq \rho_{n} \frac{\mu}{\rho_{n+1}}\left\|y_{n}-\omega_{n}\right\| .
\end{aligned}
$$

So,

$\left\|x_{n+1}-\omega_{n}\right\| \leq\left\|x_{n+1}-y_{n}\right\|+\left\|y_{n}-\omega_{n}\right\| \leq\left(1+\rho_{n} \frac{\mu}{\rho_{n+1}}\right)\left\|y_{n}-\omega_{n}\right\|$, thus having

$$
\left\|y_{n}-\omega_{n}\right\| \geq \frac{1}{\left(1+\rho_{n}\left(\mu / \rho_{n+1}\right)\right)}\left\|x_{n+1}-\omega_{n}\right\| .
$$

Let $p \in V I(C, \mathfrak{I})$, through Theorem 1 , and we can get

$$
\left\|x_{n+1}-p\right\|^{2} \leq\left\|\omega_{n}-p\right\|^{2}-\left(1-\rho_{n}^{2} \frac{\mu^{2}}{\rho_{n+1}^{2}}\right)\left\|y_{n}-\omega_{n}\right\|^{2} \text {. }
$$

From (23) and (24), we can get

$$
\begin{aligned}
\left\|x_{n+1}-p\right\|^{2} & \leq\left\|\omega_{n}-p\right\|^{2}-\frac{1-\rho_{n}^{2}\left(\mu^{2} / \rho_{n+1}^{2}\right)}{1+\rho_{n}^{2}\left(\mu^{2} / \rho_{n+1}^{2}\right)}\left\|x_{n+1}-\omega_{n}\right\|^{2} \\
& =\left\|\omega_{n}-p\right\|^{2}-\frac{1-\rho_{n}\left(\mu / \rho_{n+1}\right)}{1+\rho_{n}\left(\mu / \rho_{n+1}\right)}\left\|x_{n+1}-\omega_{n}\right\| \\
& \leq\left\|\omega_{n}-p\right\|^{2}-\mathfrak{\Re}\left\|x_{n+1}-\omega_{n}\right\|^{2} .
\end{aligned}
$$

Through the construction of $\omega_{n}$, we can get

$$
\begin{aligned}
\left\|\omega_{n}-p\right\|^{2}= & \left\|x_{n}+\eta_{n}\left(x_{n}-x_{n-1}\right)-p\right\| \\
= & \left\|\left(1+\eta_{n}\right)\left(x_{n}-p\right)-\eta_{n}\left(x_{n-1}-p\right)\right\| \\
\leq & \left(1+\eta_{n}\right)\left\|x_{n}-p\right\|^{2}-\eta_{n}\left\|x_{n-1}-p\right\|^{2} \\
& +\eta_{n}\left(1+\eta_{n}\right)\left\|x_{n}-x_{n-1}\right\|^{2} .
\end{aligned}
$$

From (25) and (26), we can get

$$
\begin{aligned}
\left\|x_{n+1}-p\right\|^{2} & \leq\left(1+\eta_{n}\right)\left\|x_{n}-p\right\|^{2}-\eta_{n}\left\|x_{n-1}-p\right\|^{2}+\eta_{n}\left(1+\eta_{n}\right)\left\|x_{n}-x_{n-1}\right\|^{2} \\
& \leq\left(1+\eta_{n}\right)\left\|x_{n}-p\right\|^{2}-\eta_{n}\left\|x_{n-1}-p\right\|^{2}+2 \eta\left\|x_{n}-x_{n-1}\right\|^{2} .
\end{aligned}
$$

On the other hand,

$$
\begin{aligned}
\left\|x_{n+1}-\omega_{n}\right\|^{2} & =\left\|x_{n+1}-x_{n}-\eta_{n}\left(x_{n}-x_{n-1}\right)\right\|^{2} \\
& =\left\|x_{n+1}-x_{n}\right\|^{2}+\eta_{n}^{2}\left\|x_{n}-x_{n-1}\right\|^{2}-2 \eta_{n}\left\langle x_{n+1}-x_{n}, x_{n}-x_{n-1}\right\rangle \\
& \geq\left\|x_{n+1}-x_{n}\right\|^{2}+\eta_{n}^{2}\left\|x_{n}-x_{n-1}\right\|^{2}-2 \eta_{n}\left\|x_{n+1}-x_{n}\right\|\left\|x_{n}-x_{n-1}\right\| \\
& \geq\left(1-\eta_{n}\right)\left\|x_{n+1}-x_{n}\right\|^{2}+\left(\eta_{n}^{2}-\eta_{n}\right)\left\|x_{n}-x_{n-1}\right\|^{2} .
\end{aligned}
$$


From (25), (26), and (28), we get

$$
\begin{aligned}
\left\|x_{n+1}-p\right\|^{2} \leq & \left(1+\eta_{n}\right)\left\|x_{n}-p\right\|^{2}-\eta_{n}\left\|x_{n-1}-p\right\|^{2}+\eta_{n}\left(1+\eta_{n}\right)\left\|x_{n}-x_{n-1}\right\|^{2} \\
& -\mathfrak{\Re}\left(1+\eta_{n}\right)\left\|x_{n+1}-x_{n}\right\|^{2}-\mathfrak{\Re}\left(\eta_{n}^{2}-\eta_{n}\right)\left\|x_{n}-x_{n-1}\right\|^{2} \\
= & \left(1+\eta_{n}\right)\left\|x_{n}-p\right\|^{2}-\eta_{n}\left\|x_{n-1}-p\right\|^{2}-\Re\left(1-\eta_{n}\right)\left\|x_{n+1}-x_{n}\right\|^{2} \\
& +\left[\eta_{n}\left(1+\eta_{n}\right)-\mathfrak{\Omega}\left(\eta_{n}^{2}-\eta_{n}\right)\right]\left\|x_{n}-x_{n-1}\right\|^{2} \\
= & \left(1+\eta_{n}\right)\left\|x_{n}-p\right\|^{2}-\eta_{n}\left\|x_{n-1}-p\right\|^{2}-\gamma_{n}\left\|x_{n+1}-x_{n}\right\|^{2}+\mu_{n}\left\|x_{n}-x_{n-1}\right\|^{2},
\end{aligned}
$$

where $\quad \gamma_{n}:=\mathfrak{\Re}\left(1-\eta_{n}\right) \quad$ and $\quad \mu_{n}:=\eta_{n}\left(1+\eta_{n}\right)-\mathfrak{\Re}$ $\left(\eta_{n}^{2}-\eta_{n}\right) \geq 0$.

Let $\Gamma_{n}:=\left\|x_{n}-p\right\|^{2}-\eta_{n}\left\|x_{n-1}-p\right\|^{2}+\mu_{n}\left\|x_{n}-x_{n-1}\right\|^{2}$. From (29), we can obtain

$$
\begin{aligned}
\Gamma_{n+1}-\Gamma_{n}= & \left\|x_{n+1}-p\right\|^{2}-\left(1+\eta_{n+1}\right)\left\|x_{n}-p\right\|^{2}+\eta_{n}\left\|x_{n-1}-p\right\|^{2} \\
& +\mu_{n+1}\left\|x_{n+1}-x_{n}\right\|^{2}-\mu_{n}\left\|x_{n}-x_{n-1}\right\|^{2} \\
\leq & \left\|x_{n+1}-p\right\|^{2}-\left(1+\eta_{n}\right)\left\|x_{n}-p\right\|^{2}+\eta_{n}\left\|x_{n-1}-p\right\|^{2} \\
& +\mu_{n+1}\left\|x_{n+1}-x_{n}\right\|^{2}-\mu_{n}\left\|x_{n}-x_{n-1}\right\|^{2} \\
\leq & -\left(\gamma_{n}-\mu_{n+1}\right)\left\|x_{n+1}-x_{n}\right\|^{2} .
\end{aligned}
$$

Because $0 \leq \eta_{n} \leq \eta_{n+1} \leq \eta$, so

$$
\begin{aligned}
\gamma_{n}-\mu_{n+1} & =\mathfrak{\Re}\left(1-\eta_{n}\right)-\eta_{n+1}\left(1+\eta_{n+1}\right)+\mathfrak{\Re}\left(\eta_{n+1}^{2}-\eta_{n+1}\right) \\
& \geq \mathfrak{\Re}\left(1+\eta_{n+1}\right)-\eta_{n+1}\left(1+\eta_{n+1}\right)+\mathfrak{\Re}\left(\eta_{n+1}^{2}-\eta_{n+1}\right) \\
& \geq \mathfrak{\Re}\left(1-\eta_{n}\right)-\eta(1+\eta)+\mathfrak{\Re}\left(\eta^{2}-\eta\right) \\
& \geq-(1-\mathfrak{\Re}) \eta^{2}-(1+2 \mathfrak{\Re}) \eta+\mathfrak{\Re} .
\end{aligned}
$$

Combining (30) and (31), we get

$$
\Gamma_{n+1}-\Gamma_{n} \leq-\delta\left\|x_{n+1}-x_{n}\right\|^{2}
$$

where $\delta:=-(1-\mathfrak{K}) \eta^{2}-(1+2 \mathfrak{K}) \eta+\mathfrak{K}, \delta>0$ can be obtained from (20).

Easy to prove

$$
\Gamma_{n+1}-\Gamma_{n} \leq 0 .
$$

Therefore, the sequence $\left\{\Gamma_{n}\right\}$ is nonincreasing.

On the other hand, due to $\mu_{n} \geq 0$, there is

$$
\begin{aligned}
\Gamma_{n} & =\left\|x_{n}-p\right\|^{2}-\eta_{n}\left\|x_{n-1}-p\right\|^{2}+\mu_{n}\left\|x_{n}-x_{n-1}\right\|^{2} \\
& \geq\left\|x_{n}-p\right\|^{2}-\eta_{n}\left\|x_{n-1}-p\right\|^{2} .
\end{aligned}
$$

This means

$\left\|x_{n}-p\right\|^{2} \leq \eta_{n}\left\|x_{n-1}-p\right\|^{2}+\Gamma_{n} \leq \eta\left\|x_{n-1}-p\right\|^{2}+\Gamma_{1} \leq \cdots \leq \eta^{n}\left\|x_{0}-p\right\|^{2}+\Gamma_{1}\left(1+\cdots+\eta^{n-1}\right) \leq \eta^{n}\left\|x_{0}-p\right\|^{2}+\frac{\Gamma_{1}}{1-\eta}$

Similarly,

$$
\begin{aligned}
\Gamma_{n+1} & =\left\|x_{n+1}-p\right\|^{2}-\eta_{n+1}\left\|x_{n}-p\right\|^{2}+\mu_{n+1}\left\|x_{n+1}-x_{n}\right\|^{2} \\
& \geq\left\|x_{n+1}-p\right\|^{2}-\eta_{n+1}\left\|x_{n}-p\right\|^{2} \geq-\eta_{n+1}\left\|x_{n}-p\right\|^{2} .
\end{aligned}
$$

From (35) and (36), we can see that

$$
-\Gamma_{n+1} \leq \eta_{n+1}\left\|x_{n}-p\right\|^{2} \leq \eta\left\|x_{n}-p\right\|^{2} \leq \eta^{n+1}\left\|x_{0}-p\right\|^{2}+\frac{\eta \Gamma_{1}}{1-\eta} .
$$

According to (32), we get the following inequality:

$$
\begin{aligned}
\delta \sum_{n=1}^{k}\left\|x_{n+1}-x_{n}\right\|^{2} & \leq \Gamma_{1}-\Gamma_{k+1} \leq \eta^{k+1}\left\|x_{0}-p\right\|^{2}+\frac{\Gamma_{1}}{1-\eta} \\
& \leq\left\|x_{0}-p\right\|^{2}+\frac{\Gamma_{1}}{1-\eta}
\end{aligned}
$$

In other words, $\quad \sum_{n=1}^{+\infty}\left\|x_{n+1}-x_{n}\right\|^{2}<+\infty$, $\left\|x_{n+1}-x_{n}\right\|^{2} \longrightarrow 0$ can be obtained from the convergence series property, that is, $\left\|x_{n+1}-x_{n}\right\| \longrightarrow 0$. So,

$$
\begin{aligned}
\left\|x_{n+1}-\omega_{n}\right\|^{2}= & \left\|x_{n+1}-x_{n}\right\|^{2}+\eta_{n}^{2}\left\|x_{n}-x_{n-1}\right\|^{2} \\
& -2 \eta_{n}\left\langle x_{n+1}-x_{n}, x_{n}-x_{n-1}\right\rangle .
\end{aligned}
$$

So, $\left\|x_{n+1}-\omega_{n}\right\| \longrightarrow 0$. By (27) and Lemma 3, we have 


$$
\lim _{n \longrightarrow+\infty}\left\|x_{n}-p\right\|^{2}=l
$$

and from (26), we can get

$$
\begin{aligned}
\left\|\omega_{n}-p\right\|^{2}= & \left\|x_{n}-p\right\|^{2}+\eta_{n}\left(\left\|x_{n}-p\right\|^{2}-\left\|x_{n-1}-p\right\|^{2}\right) \\
& +\eta_{n}\left(1+\eta_{n}\right)\left\|x_{n}-x_{n-1}\right\|^{2} .
\end{aligned}
$$

According to the sequence, $\left\{\eta_{n}\right\}$ is bounded, so

$$
\lim _{n \longrightarrow+\infty}\left\|\omega_{n}-p\right\|^{2}=l \text {. }
$$

From this, we get that $\left\{x_{n}\right\},\left\{\omega_{n}\right\}$, and $\left\{z_{n}\right\}$ are all bounded sequences, and then there

$$
0 \leq\left\|x_{n}-\omega_{n}\right\| \leq\left\|x_{n}-x_{n+1}\right\|+\left\|x_{n+1}-\omega_{n}\right\| \longrightarrow 0, n \longrightarrow+\infty .
$$

Now, we prove that the sequence $\left\{x_{n}\right\}$ weakly converges to $p \in V I(C, \mathfrak{I})$. In fact, because the sequence $\left\{x_{n}\right\}$ is bounded, we assume that there is a subset of the sequence $\left\{x_{n}\right\}$, which makes it weakly converge to $z \in H$. Without loss of generality, we still use $\left\{x_{n}\right\}$ below to represent the subset. That is, $x_{n} \rightarrow z$, where " $\rightarrow$ " represents weak convergence.

Because of $\left\|x_{n}-\omega_{n}\right\| \longrightarrow 0$, there is a subset to make it weakly converge to $z$. We still use $\left\{\omega_{n}\right\}$ below to represent the sequence, which is $\omega_{n} \rightarrow z$. Similarly, the following uses $\left\{y_{n}\right\}$ to represent the subset of the original number sequence $\left\{y_{n}\right\}$. Since $\mathfrak{T}$ is a monotone operator and $y_{n}=P_{C}\left(\omega_{n}-\rho_{n} \mathfrak{T} \omega_{n}\right)$, $\forall x \in C$, there is

$$
\begin{aligned}
0 & \leq\left\langle y_{n}-\omega_{n}+\rho_{n} \mathfrak{T} \omega_{n}, x-y_{n}\right\rangle \\
& =\left\langle y_{n}-\omega_{n}, x-y_{n}\right\rangle+\rho_{n}\left\langle\mathfrak{T} \omega_{n}, x-y_{n}\right\rangle \\
& =\left\langle y_{n}-\omega_{n}, x-y_{n}\right\rangle+\rho_{n}\left\langle\mathfrak{T} \omega_{n}, x-\omega_{n}\right\rangle+\rho_{n}\left\langle\mathfrak{T} \omega_{n}, \omega_{n}-y_{n}\right\rangle \\
& \leq\left\langle y_{n}-\omega_{n}, x-y_{n}\right\rangle+\rho_{n}\left\langle\mathfrak{T} \omega_{n}, x-\omega_{n}\right\rangle+\rho_{n}\left\langle\mathfrak{I} x, \omega_{n}-y_{n}\right\rangle .
\end{aligned}
$$

Let $n \longrightarrow+\infty$, then $\forall x \in C$ has

$$
\langle\mathfrak{I} x, x-z\rangle \geq 0 \text {. }
$$

By Lemma 4, we can get $z \in V I(C, \mathfrak{I})$, so we have proved the following:

(a) $\forall p \in V I(C, \mathfrak{I})$, then $\lim _{n \longrightarrow \infty}\left\|x_{n}-p\right\|^{2}$ limits exist;

(b) Each weak convergence point of the logarithmic sequence $\left\{x_{n}\right\}$ is in $\operatorname{VI}(C, \mathfrak{I})$.

From Lemma 3 , we know that the sequence $\left\{x_{n}\right\}$ weakly converges to $p \in V I(C, \mathfrak{T})$.

Corollary 1. Given $\rho_{0}>0$, suppose that $\left\{x_{n}\right\}$ be a sequence in $H$ defined by

$$
\left\{\begin{array}{l}
x_{0}, x_{1} \in H, \mu \in\left(\frac{1}{\sqrt{2}}, 1\right), \\
y_{n}=P_{C}\left(x_{n}-\rho_{n} \mathfrak{T} x_{n}\right), \\
x_{n+1}=y_{n}-P_{C}\left(\rho_{n} \mathfrak{T} y_{n}-\rho_{n} \mathfrak{T} x_{n}\right),
\end{array}\right.
$$

where

$$
\rho_{n+1}= \begin{cases}\min \left\{\frac{\mu\left\|y_{n}-\omega_{n}\right\|}{\left\|\mathfrak{T} y_{n}-\mathfrak{T} \omega_{n}\right\|}, \rho_{n}\right\}, & \mathfrak{T} y_{n}-\mathfrak{T} \omega_{n} \neq 0, \\ \rho_{n}, & \text { other. }\end{cases}
$$

Then, the sequence $\left\{x_{n}\right\}$ converges weakly to an element of $\in V I(C, \mathfrak{I})$.

Lemma 9. Let $\pi>0$. A point $x \in C$ is a solution of $V I(C, \mathfrak{I})$ if and only if $x \in$ FixP $_{C}(I d-\pi \mathfrak{T})$ (see [16]).

According to Theorem 2 and Lemma 9, we get the following two inferences.

Corollary 2. Let $\left\{\rho_{n}\right\}$ be a sequence obtained by Algorithm 1, and $\pi>0$ and FixP $P_{C}(I d-\pi \mathfrak{I}) \neq \varnothing$. If $\left\{\eta_{n}\right\}$ is a nonincreasing sequence, make $0 \leq \eta_{n} \leq \eta$ and $\eta<(\sqrt{1+8 \Re-}$ $1-2 \mathfrak{K}) /(2(1-2 \mathfrak{K}))$, where $\mathfrak{K}:=1-\mu^{2}$, then the sequence $\left\{x_{n}\right\}$ obtained by Algorithm 1 weakly converges to FixP $P_{C}$ $(I d-\pi \mathfrak{T})$.

Corollary 3. Given $\rho_{0}>0$, suppose that $\left\{x_{n}\right\}$ be a sequence in $H$ defined by

$$
\left\{\begin{array}{l}
x_{0}, x_{1} \in H, \mu \in\left(\frac{1}{\sqrt{2}}, 1\right), \\
y_{n}=P_{C}\left(x_{n}-\rho_{n} \mathfrak{T} x_{n}\right), \\
x_{n+1}=y_{n}-P_{C}\left(\rho_{n} \mathfrak{T} y_{n}-\rho_{n} \mathfrak{T} x_{n}\right),
\end{array}\right.
$$

where

$$
\rho_{n+1}= \begin{cases}\min \left\{\frac{\mu\left\|y_{n}-\omega_{n}\right\|}{\left\|\mathfrak{T} y_{n}-\mathfrak{T} \omega_{n}\right\|}, \rho_{n}\right\}, & \mathfrak{T} y_{n}-\mathfrak{T} \omega_{n} \neq 0, \\ \rho_{n}, & \text { other. }\end{cases}
$$

Then, the sequence $\left\{x_{n}\right\}$ converges weakly to an element of $\operatorname{Fix} P_{C}(I d-\pi \mathfrak{T})$.

The new Tseng-like gradient method is convergent. It is known that the algorithm has good accuracy due to the selection of step size. Finally, it also can be known that the algorithm has good applicability through inference. 


\section{Data Availability}

The data used to support the findings of this study are available from the corresponding author upon request.

\section{Conflicts of Interest}

The authors declare that they have no conflicts of interest.

\section{Acknowledgments}

This work was supported by the grants study on numerical simulation of soil solute transport based on lattice Boltzmann method project of the National Natural Science Foundation of China 11861003. Professor Hefang Jing is the project host and the project funding is $400,000 \mathrm{RMB}$.

\section{References}

[1] Y. I. Alber and A. N. Iusem, "Extension of subgradient techniques for nonsmooth optimization in Banach spaces," Set-Valued Analysis, vol. 9, no. 4, pp. 315-335, 2001.

[2] F. Alvarez, "Weak convergence of a relaxed and inertial hybrid projection-proximal point Algorithm for maximal monotone operators in Hilbert space," SIAM Journal on Optimization, vol. 14, no. 3, pp. 773-782, 2004.

[3] H. Attouch, X. Goudou, and P. Redont, "The heavy ball with friction method, I. The continuous dynamical system: global exploration of the local minima of a real-valued function by asymptotic analysis of a dissipative dynamical system," Communications in Contemporary Mathematics, vol. 2, no. 1, pp. 1-34, 2000.

[4] H. Attouch and M.-O. Czarnecki, "Asymptotic control and stabilization of nonlinear oscillators with non-isolated equilibria," Journal of Differential Equations, vol. 179, no. 1, pp. 278-310, 2002.

[5] H. H. Bauschke and P. L. Combettes, Convex Analysis and Monotone Operator Theory in Hilbert Spaces, Springer, New York, NY, USA, 2011.

[6] R. I. Bot, E. R. Csetnek, and S. C. Laszlo, "An inertial forwardbackward algorithm for the minimization of the sum of two nonconvex functions," EURO Journal on Computational Optimization, vol. 4, pp. 3-25, 2016.

[7] R. I. Bot and E. R. Csetnek, "An inertial Tsengs type proximal algorithm for nonsmooth and nonconvex optimization problems," Journal of Optimization Theory and Applications, vol. 171, pp. 600-616, 2015.

[8] R. I. Bot, E. R. Csetnek, and C. Hendrich, "Inertial DouglasRachford splitting for monotone inclusion problems," Applied Mathematics and Computation, vol. 256, pp. 472-487, 2015.

[9] R. I. Bot and E. R. Csetnek, "An inertial alternating direction method of multipliers," Minimax Theory and Applications, vol. 1, pp. 29-49, Springer, Dordrecht, Netherlands, 2016.

[10] R. I. Bot and E. R. Csetnek, "A hybrid proximal-extragradient algorithm with inertial effects?” Numerical Functional Analysis and Optimization, vol. 36, no. 8, pp. 951-963, 2015.

[11] Y. Censor, A. Gibali, and S. Reich, "Algorithms for the split variational inequality problem," Numerical Algorithms, vol. 59, no. 2, pp. 301-323, 2012.

[12] D. V. Thong and D. V. Hieu, "Modified Tseng's extragradient algorithms for variation inequality problems," Journal of Fixed Point Theory and Applications, vol. 20, no. 4, 2018.
[13] R. I. Bot and E. R. Csetnek, "An inertial forward-backwardforward primal-dual splitting algorithm for solving monotone inclusion problems," Numerical Algorithms, vol. 71, pp. 519540, 2016.

[14] F. Alvarez and H. Attouch, "An inertial proximal method for maximal monotone operators via discretization of a nonlinear oscillator with damping," Set-Valued Analysis, vol. 9, no. 1-2, pp. 3-11, 2001.

[15] W. Takahashi, Nonlinear Functional Analysis; Fixed Point Theory and its Applications, Yokohama Publishers, vol. 152, 18 pages, Yokohama, Tokyo, Japan, 2000.

[16] L. J. Zhu, Y. Yao, and M. Postolache, "Projection methods with linesearch technique for pseudomonotone equilibrium problems and fixed point problems," UPB Scientific Bulletin, Series A, In press. 